\title{
Acute Care SINS: Surgical Insights for the Non-surgeon Chapter 9: Urology SINS
}

Adam Kinnaird MD PhD, Adrian Fairey MD MSc, Keith Rourke MD, Rachel G. Khadaroo MD PhD, Peter G. Brindley MD

\section{About the Authors}

Adam Kinnaird is a resident in urology; Adrian Fairey is an assistant professor of surgery; Rachel Khadaroo is an assistant professor of surgery and a member of the Divisions of Critical Care Medicine and General Surgery; Keith Rourke is an associate professor of surgery; and Peter Brindley is a professor of critical care medicine, all at the University of Alberta, in Edmonton, Alberta. Correspondence may be directed to

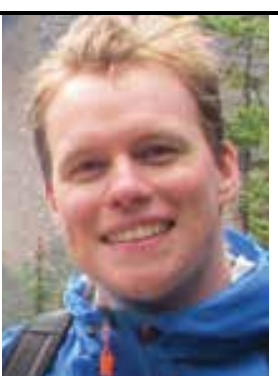

Kinnaird

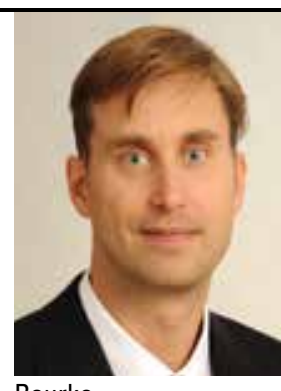

Rourke

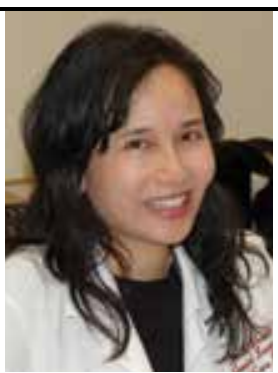

Khadaroo

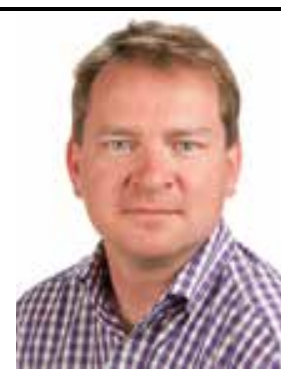

Brindley peter.brindley@albertahealthservices.ca.

\begin{abstract}
Summary
"Surgical Insights for the Non-surgeon," or SINS, is composed of several short chapters intended to cover fundamental surgical knowledge for non-surgeons. The authors focus on surgical pearls, operative insights, and applied anatomy. In Chapter 9 of this series, the authors address the genitourinary system and Urology SINS.
\end{abstract}

\section{Résumé}

L'ouvrage «Surgical Insights for the Non-surgeon » (aperçu de la chirurgie à l'intention du non-chirurgien) se compose de courts chapitres couvrant les connaissances fondamentales en chirurgie. Les auteurs se concentrent sur des enseignements tirés de leur expérience, des aspects opératoires et l'anatomie appliquée. Le neuvième chapitre porte sur le système génitourinaire et l'urologie.

He who works with his hands is a laborer; he who works with his head and hands is a craftsman.

$$
\text { - Francis of Assisi }
$$

\section{Anatomy}

Urology is plumbing! Starting from the top of the genitourinary tract, urine is first concentrated in the collecting ducts of the renal medulla. From there, urine flows into the renal collecting system by passing from collecting duct to minor calyx, to major calyx, to renal pelvis, down the ureter, and into the bladder for storage. The fornix of a minor calyx can rupture (almost like a pressure-release valve) following severe obstruction (typically a ureteric stone). However, calyceal rupture is not an indication for emergent surgery.
The ureters run along the psoas muscle, cross over the iliac vessels at their bifurcation, and enter the posterior aspect of the bladder on opposite ends of the trigone muscle. The ureter is not the only tubular structure in the pelvis; the gonadal veins and the appendix also share that distinction. The bladder is an extraperitoneal muscular reservoir tucked behind the pubic symphysis. Continence relies upon the synergic relaxation of detrusor muscles and the simultaneous contraction of the bladder neck and pelvic floor muscles. Typically, urine does not reflux up a ureter, owing to a one-way flap valve created 
as the ureteral orifice is compressed while the bladder fills. The most common places for ureteric calculi are the ureteropelvic junction, the crossing of the iliac vessels, and the ureterovesical junction (UVJ). The UVJ is the most distal portion of the ureter, and it also happens to be the narrowest. The UVJ is where most stones get "stuck."

The base of the bladder opens into the urethra, like the drain of a sink. When empty, the bladder is about the size and shape of a pear; when full, the typical adult bladder's capacity is approximately $500 \mathrm{cc}$. A child's bladder capacity is estimated by the following formula:

$$
(\text { age }+2) \times 30 c c
$$

For example, the bladder capacity of a 4-year-old is determined as follows:

$$
(4+2) \times 30 c c=180 c c
$$

The bladder's inside is covered by a watertight mucosal surface made up of transitional epithelial cells. These are the cells that most commonly transform into a bladder cancer.

The urethra has five anatomical sections: prostatic, membranous, bulbar, penile, and fossa navicularis. The prostate is a walnut-sized, conically shaped gland with four lobes. It is located between the bladder and the penis, just in front of the rectum (conveniently for digital palpation). It serves as a merging lane for urine and semen, which the latter enters via the ejaculatory ducts in the prostate. The urethra runs through the centre of the prostate, which is why benign prostatic hyperplasia $(\mathrm{BPH})$ results in urinary obstruction, retention, and nocturnal bathroom trips. For a variety of non-urologic reasons, however, one or two episodes of nocturia per night is normal for many people.

Because it passes through the penis, the male urethra is longer $(20 \mathrm{~cm})$ than the female urethra $(4 \mathrm{~cm})$; the shorter separation from skin flora in females is why women are more likely to experience urinary tract infections. Urethral injuries associated with pelvic fractures occur at the junction of the membranous and bulbar urethra (and in approximately 5\% of all pelvic fractures). The fossa navicularis, which is within 1 $\mathrm{cm}$ of the urethral meatus, is the narrowest part of the urethra. Therefore, if a catheter can pass the first $2 \mathrm{~cm}$ but then stops at any point, this is not due to a catheter of the wrong size; there is a pathologic reason (false passage, large prostate, urethral stricture, foreign object, etc.).

The remainder of this article discusses common reasons for urology consults.

\section{Hematuria}

- Divided into microscopic versus gross (i.e., visible) hematuria - In both cases, blood may come from any site along the genitourinary tract

- Causes include trauma, tumours (prostate, bladder, renal), $\mathrm{BPH}$, stones, radiation cystitis

- Generally, hematuria is a urologic malignancy until proven otherwise

- The urinary tract is divided into two parts for evaluation

- The upper tract (kidneys, ureters)

- The lower tract (bladder, prostate, urethra)

\section{Microscopic Hematuria}

- Defined as $>3$ red blood cells per high-powered field

- Work up completely in patients $>40$ years old

- Complete blood count (CBC)

- Serum creatinine $(\mathrm{Cr})$

- Urine microscopy and culture

- Urine cytology

- Cystoscopy

- Upper-tract imaging (ultrasound kidneys, ureters, bladder)

\section{Gross Hematuria}

- Defined as bloody urine visible to the naked eye

- Work up for patients of all ages

- CBC

- Serum Cr

- Urine culture

- Cystoscopy

- Upper-tract imaging (contrast-enhanced computed tomography of abdomen/pelvis)

- Gross hematuria can result in numerous blood clots in the bladder, which in turn can cause urinary retention (called "clot retention")

- The best way to manage clot retention is to remove all blood clots from the bladder

- Manually irrigate a three-way Foley catheter with normal saline

o To decompress the bladder and remove clots

o See below for technical instructions

- Next, follow with continuous bladder irrigation (CBI) through the irrigation (large) channel of the three-way catheter

- Titrate the CBI flow to keep the urine clear

- If blockage reoccurs, manually irrigate again

- Remember, only manual irrigation clears bladder clots; CBI only prevents them from re-forming

\section{Manual Irrigation Technique}

- Equipment: one 60 cc Toomey syringe, one irrigation kit, $1 \mathrm{~L}$ normal saline (NS) 
- Technique

- Place a large (22-24 French) three-way Foley catheter

- Instill 60 cc of NS through the middle (large) port, using a Toomey syringe

- Follow immediately with vigorous aspiration through the same port

- Repeat until $1 \mathrm{~L}$ of NS is used or the urine is clear

- If you can instill fluid but cannot aspirate

- The catheter is against the bladder wall or malpositioned (i.e., not in the bladder!)

- If so, instill $120 \mathrm{cc}$ into the bladder prior to aspiration

- If you still cannot aspirate, the catheter is likely malpositioned

o So stop! Deflate the balloon and remove the catheter

o Then call urology

\section{Troubleshooting the Difficult Urethral Catheter General Tips}

- Urinary catheters are measured in "French," abbreviated as "F"

- "French" refers to the French scale, or French gauge

- French is a measure of the external circumference of a catheter in millimetres (for example, a 16F catheter has an external circumference of $16 \mathrm{~mm}$ )

- Catheters range from $8 \mathrm{~F}$ (pediatric) to $24 \mathrm{~F}$

- The typical adult catheter measures $14 \mathrm{~F}$ to $18 \mathrm{~F}$

- Larger catheters (20F or greater) are used to irrigate a bladder or ensure that a bladder is well decompressed

- Look for urine return before inflating the catheter balloon; if you do not see urine draining, you may still be in the urethra

- If no urine returns but you believe that you are in the bladder (i.e., no urine due to renal failure or septic shock),

- Aspirate the drainage port, or try manual irrigation (as above)

- BUT, then only inflate the balloon if you see fluid return with aspiration

- That is don't inflate the balloon if fluid does not return with aspiration

-You do not need to test balloon inflation prior to insertion

- This only makes the tip larger and more uncomfortable when inserted

- Also, catheters are designed to be inflated only once

- Do not attach the sterile water syringe until the catheter is inserted; doing so only adds a useless counterweight that may pull the catheter out inadvertently

- Apply at least one tube of sterile xylocaine jelly to the urethra before insertion; if unavailable, ensure the catheter is at least well lubricated

-When inserting a catheter, make sure to "pull the penis to the sky" to straighten the angle between the penile urethra and bulbar urethra
- Always return the foreskin back over the glans to prevent paraphimosis (see below)

- Bacterial colonization of an indwelling catheter is approximately $10 \%$ per day

- Expect most patients to have (at least) asymptomatic bacteriuria by 1 week

- Remove the catheter when no longer required

\section{Difficult Catheters}

- Difficulty with placement may be due to an enlarged prostate, urethral stricture, false passage, phimosis, bladder neck contracture, or hypospadias

- A "false passage" is a tear in the urethral epithelium

- Due to failed (usually multiple) attempts

- The catheter enters under the flap

- Causing a false passage and creating a one-way valve

o Therefore you can push fluid in, but cannot aspirate fluid out

- Catheters may not pass due to an enlarged prostate or a steep angle between an enlarged prostate and the bladder

- In such a case, the catheter may pass but not all the way to the bladder

- Try a larger $(18 \mathrm{~F}$ or $20 \mathrm{~F})$ catheter to push the prostate lobes "aside," or try a coudé-tip catheter (see below)

- If you suspect a false passage, use a coudé-tip catheter

- A urethral stricture is scar tissue or fibrosis that narrows the urethra

- Suspect a urethral stricture if the catheter passes initially but not as far as the prostate

- Try a smaller ( $12 \mathrm{~F}$ or $14 \mathrm{~F})$ catheter to try to "sneak" through the small urethral lumen

- A bladder neck contracture is scarring of the prostatic urethra, usually occurring after prostate surgery

-When placing a urinary catheter, the non-urologist should

- Try a regular $16 \mathrm{~F}$ or $18 \mathrm{~F}$ catheter with lots of lubrication and while keeping the penis stretched

- If this fails, try a larger ( $18 \mathrm{~F}$ or $20 \mathrm{~F})$ coudé-tip catheter to bypass a false passage or to push the prostate out of the way

- If the above two steps fail, try a smaller catheter (12F or $14 \mathrm{~F})$

o A stricture could be the culprit

o A smaller catheter may "negotiate" the narrowed urethra

- If the above three steps fail, call urology

- Sometimes a catheter that doesn't drain urine may be in the bladder, but the patient is anuric

- When in doubt, irrigate the catheter

- Then obtain a serum creatinine level, to check renal function

- Also, do an ultrasound examination of the bladder to confirm that urine is present 


\section{Coudé-Tip Catheters}

- A catheter (often coloured red) that is bent like a hockey stick at the end

- This catheter mimics the upward curve at the junction of the anterior and posterior urethra

- This catheter can aid passage through the prostatic urethra

- False passages usually occur posteriorly, and the coudé curve can bypass these; the curve causes the tip to pass over (but not into) the false passage

- The curved tip should always be pointing up

- Once the catheter is inside the urethra, you can no longer see the tip

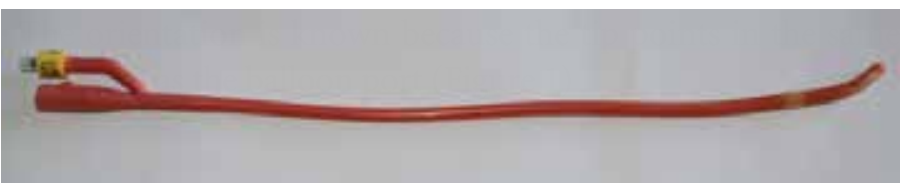

Figure 1. Coudé tip catheter.

The orientation of the catheter is known because the tip points the same direction as the balloon port.

\section{Three-Way Catheters}

- Colloquially called CBI catheters or "hematuria catheters"

- Sizes are 20F to 24F; typically, bigger = better for draining clots

- If indicated (i.e., gross hematuria), the technique is the same as with a usual catheter

- Manually irrigate through the middle (large) port, as described above (Figure 2)

- Connect inflow port (on the side opposite the balloon port) to a $4 \mathrm{~L}$ bag of NS

- Proceed with CBI (as above)

- Note: You will no longer be able to accurately track urine output while CBI is running

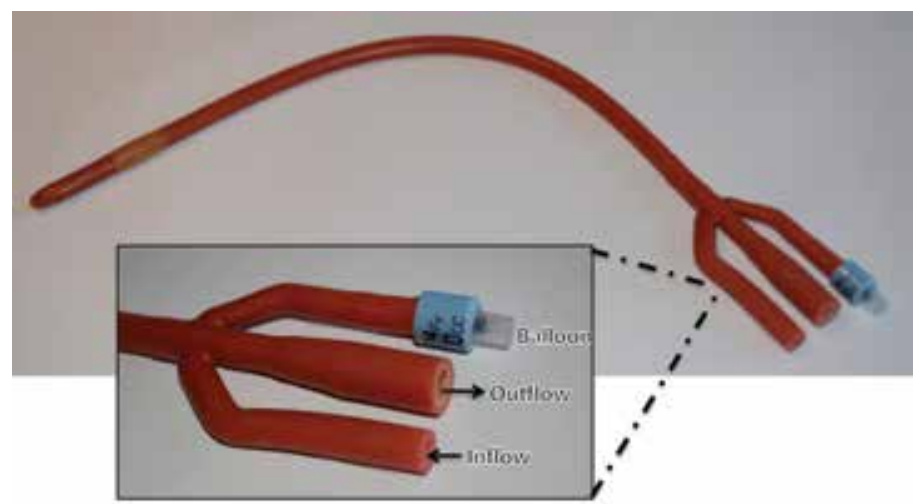

Figure 2. Three-way catheter.

Photos by Keith Rudolf

\section{Suprapubic Catheters}

- Placed directly through the abdominal wall into the bladder
- Typically used when a urethral catheter cannot be placed (i.e., a very difficult urethral stricture) or in some patients who require long-term ( $>3$ months) catheterization

- Materials: local anaesthetic (with epinephrine), 22-gauge "seeker" needle, $10 \mathrm{cc}$ syringe, suprapubic catheter kit, urometer - Placement technique

1. Prepare a wide sterile field

- Top: level with the umbilicus

- Bottom: below the pubic symphysis

- Sides: lateral aspects of the rectus sheath

2. Landmark two fingerbreadths in midline and above the pubic symphysis

3. Inject local anaesthetic into skin, subcutaneous tissues, and down to fascia

4. Using a seeker needle, advance into the anaesthetized tract until urine is aspirated

5. Make a 2-cm stab incision down to fascia

6. Nick the fascia with the blade

7. Hold the suprapubic trochar so that your index finger acts as a guard

8. Twist the trochar through the fascia and into the bladder

9. Once urine flows, advance $1 \mathrm{~cm}$ more

10. Detach the trochar, leaving a hollow plastic sheath in situ

11. Insert a $16 \mathrm{~F}$ Foley catheter through the plastic sheath into the bladder

12. Inflate the Foley balloon prior to removing the plastic sheath

13. Withdraw the sheath, but do not to pull the Foley with it

14. Suture the skin with interrupted 3-0 Prolene superior to the Foley catheter

15. Using this same closing stitch, secure the catheter as you would any drain

- Maintenance: Leave the catheter in for 4 weeks, prior to exchanging, in order for the tract to mature

\section{Drainage Tubes}

- Obstruction or stasis of urine in the collecting systems can cause renal failure or infection

- Therefore, urinary drainage must be maintained

- This is done via hollow plastic tubes

- An enlarged prostate requires a urethral catheter (as above)

- An infected ureteric calculus requires a ureteral (ureteric) stent or nephrostomy tube

- A ureteric injury requires a nephrostomy tube

\section{Ureteral Stents}

- Commonly called "double-J" stents

- Usually 24-26 cm long, 6F-8F hollow plastic tubes 
- They create a connection between the renal pelvis and the bladder, bypassing any obstruction along the length of the ureter

- At both ends, the stent curls in a pigtail (to hold it in place)

- Stents can be introduced in both directions

- Retrograde (via cystoscopy [i.e., from the bladder up])

- Antegrade (percutaneous [i.e., from the kidney down])

- Regardless of the direction, they are inserted over a wire, with a Seldinger-like technique

- Important to note that stenting rarely treats the underlying cause; rather, it temporarily alleviates the obstruction

- Postinsertion abdominal radiography is used to determine placement

- Proper placement is when the lower pigtail is in the bladder and the upper pigtail curls in the kidney (Figure 3 )

- Note: If a patient presents with a presumed pyelonephritis and does not respond to treatment after 48 hours

- Obtain urgent renal imaging to rule out an obstructing ureteral stone

- This requires urgent decompression (i.e., stent) because acute obstructing ureteral calculus plus concurrent pyelonephritis is life-threatening

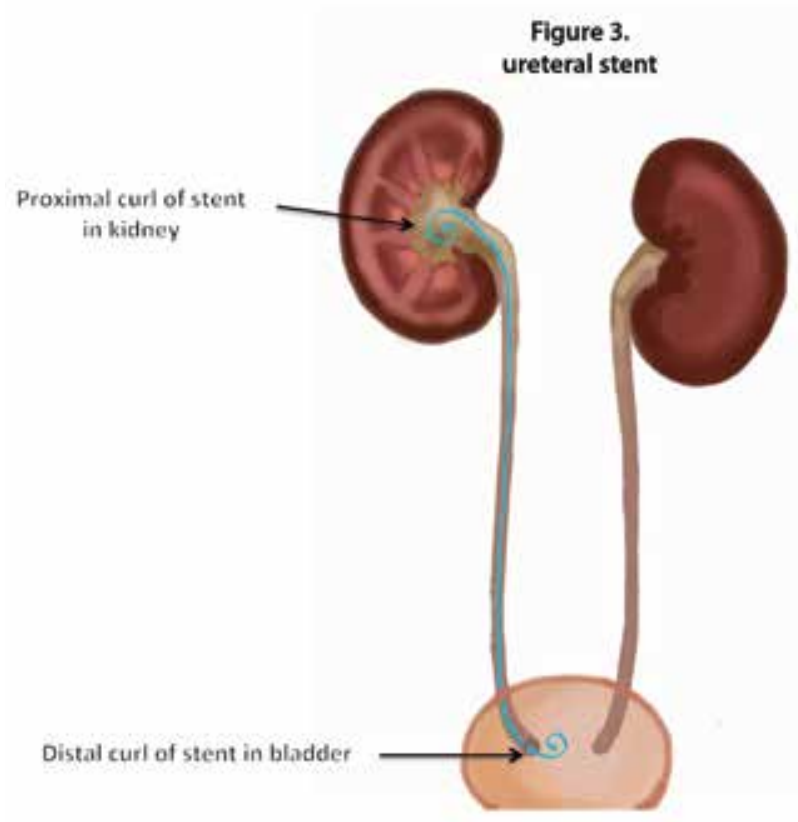

Illustration by Rachel G. Khadaroo

Figure 3. Ureteral stent.

\section{Nephrostomy Tubes}

- A percutaneous urinary diversion

- Connects the renal pelvis to a bag on the patient's back

- Used to emergently relieve obstruction and when a retrograde ureteric stent cannot be placed

- In Europe, nephrostomy tubes are the first-line treatment for infected ureteral stones; in North America, it is usually retrograde stenting (see above)

- This indicates that stenting and nephrostomy tubes are merely two ways to decompress the genitourinary system

- The most important points are that decompression is required and that either method works

- Nephrostomy tubes can be used prior to placing antegrade ureteric stents; once antegrade stents are placed, the nephrostomy tubes are typically removed within 24 hours

\section{Urinary Diversions}

- After cystectomy (bladder removal), restoration of urinary flow is needed

- To accomplish this, the ureters can be diverted to one of the following

- An ostomy made of a 10-15 cm isolated segment of ileum (such as an ileal conduit)

- A new intracorporal storage container made up of $60 \mathrm{~cm}$ of ileum (aka a neobladder)

\section{Ileal Conduit}

- A cutaneous "incontinent" urinary diversion

- Made by removing the ureters from the bladder and reconnecting them to a loop of $10-15 \mathrm{~cm}$ of detached bowel (also called an intentional ureteroenteric fistula brought out to the skin)

- Jejunum or colon can be used, but the ileum is simplest and most common

- The end of the bowel is brought through a stoma to the outside abdominal wall

- Urine drains continually into an ostomy bag

- Resorption of urine can occur via the the bowel segment; this can cause hyperchloremic/nonanion-gap metabolic acidosis

- Postoperatively, patients have temporary stents in each ureter

- This maintains patency and allows healing

- These stents are visible (i.e., they stick out of the stoma)

- The duration of ureteric stenting is variable but typically ranges from 1 to 4 weeks

- The most common long-term complication is acute pyelonephritis, which occurs in approximately $18 \%$ of patients 


\section{Neobladders}

- An orthotopic (i.e., grafted into the natural position) urinary diversion

- A new urinary reservoir is created and is connected with the native urethra

- The urethral sphincter is preserved; therefore, continence is maintained in $80-90 \%$ of cases

- A neobladder is made from $\sim 60-75 \mathrm{~cm}$ of ileum detubularized and folded into a sphere

- Ureters are anastomosed to the neobladder and stented for 1-4 weeks (as above)

- Because they end in the neobladder, these stents may not be visible

- The patient will have a urethral catheter immediately postoperation

- Neobladder irrigation is critical to maintain function, due to the buildup of mucus secreted by the ileum

- While the patient is in hospital, irrigation should be done four times daily via the indwelling urethral catheter, using $60 \mathrm{cc}$ of NS

- Perioperative complications are $20-30 \%$ higher as compared to ileal conduits, but neobladders improve long-term quality of life

- Neobladder leaks require percutaneous drainage and nephrostomy tubes

- Long-term complications include

- Vitamin $B_{12}$ deficiency (due to the large amount of terminal ileum used)

- Bladder stones (both symptomatic and asymptomatic)

\section{Unwanted Erection}

\section{Priapism}

- Defined as (1) an erection lasting more than 4 hours beyond orgasm or (2) an erection unrelated to sexual stimulation

- An emergency that, if untreated, leads to corporeal fibrosis, permanent erectile dysfunction, and (in extreme cases) penile necrosis

- Subdivided into (1) ischemic, (2) stuttering, and (3) nonischemic

- Ischemic priapism

o Analogous to compartment syndrome

o Occlusion of venous outflow that subsequently prevents arterial inflow

o Common causes include erectile dysfunction medications, hematologic dyscrasias, neurogenic injury, and recreational drugs

- Stuttering priapism

- Occurs most often in sickle cell disease o Usually self-resolving but can lead to ischemic priapism

o Recurs more frequently_ and for longer—as patients age

- Can respond to hydration

- Non-ischemic priapism

- Also known as high-flow priapism

o Caused by straddle trauma and laceration of a cavernosal artery

o Painless due to good vascular supply; therefore, low risk of permanent injury

- A corporal blood gas (i.e., a penile blood gas) is sometimes required to differentiate between ischemic and non-ischemic priapism

- Non-ischemic priapism has a blood gas profile most similar to arterial blood, whereas ischemic priapism will have a partial oxygen pressure $(\mathrm{PO} 2)<30$ and $\mathrm{pH}<7.25$

\section{Corporal Blood Gas Technique}

- Insert an 18-gauge needle at either the 3- or 9-o'clock position into the corpus cavernosum, just distal to the penoscrotal junction

- This position avoids nerves running along the dorsum of the shaft

- Pinch the penile base between two fingers, and aspirate blood from the shaft

- Typical corporal blood gas findings in ischemic priapism

$-\mathrm{PO} 2<30$

- Partial carbon dioxide pressure $(\mathrm{PCO} 2)>60$

- $\mathrm{pH}<7.25$

\section{Treatment of Ischemic Priapism}

- Use the same initial technique used to assess corporal blood gas

- Attach an 18-gauge needle to a $10 \mathrm{cc}$ syringe; aspirate the shaft until soft while pinching off the blood supply at the base of the penis

-With the needle still in place

- Irrigate (with cold NS)

- Use the same volume of NS as the volume of blood that was removed

- Repeat three times

- If priapism persists, inject intracavernosal phenylephrine (see below)

- Dilute phenylephrine to $200 \mu \mathrm{g} / \mathrm{mL}$

o Usual phenylephrine concentration is $1 \mathrm{mg} / \mathrm{mL}(1 \mathrm{~mL}$ in a vial)

o Therefore, dilute with $4 \mathrm{~mL}$ cold NS (to create $5 \mathrm{~mL}$ in total) 
- After aspirating the shaft (as above), inject $1 \mathrm{~mL}$ of this solution into the shaft

- Release your grip at the base of the penis to allow blood to refill the penis

- Repeat this every 5 minutes, up to a maximum of 5 times - If this fails, a surgical shunt is required

o A shunt creates a vascular bypass between the corpora cavernosa and the glans penis

o The shunt is achieved via bilateral transglanular incisions

\section{Penile Prostheses}

- Surgically implanted devices are used as last-line of treatment of erectile dysfunction

- Inserted into the corpora cavernosa

- Malfunction (or simple misunderstanding) may lead to misdiagnosed priapism

- Two major classes: malleable and inflatable

- Malleable prostheses

o Semirigid tubes: bent up for coitus (and bent down otherwise)

o Penis is constantly rigid

- Inflatable prostheses

o Multicomponent devices

o Two tubes in the penis, a scrotal pump, and an abdominal fluid reservoir

o The scrotal pump permits on-demand erections and detumescence

\section{Penile Fracture}

- As the corpora cavernosa fill during erection, the blood is held by a strong collagenous outer layer called the tunica albuginea (which can withstand intracavernous pressures of up to 1,500 $\mathrm{mm} \mathrm{Hg!)}$

- A penile fracture represents rupture of the tunica albuginea

- Usually caused by "buckling": most commonly, an erect penis slips out of the partner and hits the perineum or pubis, causing a sudden bend

- Fracture occurs laterally, where the tunica albuginea is thinnest

- Patients describe a cracking sound, followed by pain, and rapid detumescence

- The appearance is described as an "eggplant" deformity (swelling, bruising, dark discolouration)

- Approximately $20 \%$ of the time, the urethra is simultaneously injured, causing profuse bleeding through the urethral meatus

- Treat with prompt surgery (within 12 hours)

- Hematoma evacuation and primary repair of the tunica

- Timely surgery reduces erectile dysfunction and preserves penile curvature

\section{Surgical Pearl: \\ Performing a Dorsal Penile Nerve Block}

- Indications: reduction of paraphimosis, dorsal slit for phimosis, circumcision

- Materials: $0.5 \%$ bupivacaine (without epinephrine),

a 22-gauge needle, a 10-cc syringe

- Technique (Figure 4):

1. Clean around the superior base of the penis and the pubic symphysis

2. Locate the correct spot

o Midline, inferior to the pubic symphysis and superior to the penile base

3. Inject bupivacaine as you plunge the needle deeper o Final depth is the posterior inferior aspect of the pubic symphysis

4. Without removing the needle, direct it to either side of the midline while injecting bupivacaine to a similar depth

Note: the dorsal penile sensory nerves run on either side of midline. This technique captures both of them with only one poke.

\section{Phimosis}

- The inability to retract the foreskin for cleaning, voiding, or sexual function

- Usually from a fibrotic band of tissue

- May result in recurrent infections (balanitis)

- May result in pain or tearing or bleeding with sexual intercourse

- Treatment may be conservative (e.g., topical corticosteroids [ $0.05 \%$ clobetasol] used in the pediatric population)

- If conservative measures fail, then surgery (dorsal slit or circumcision) is required

- Dorsal slit

- Foreskin is incised dorsally through the area of phimosis

- Usually done under local anaesthetic

- Circumcision

- Complete removal of the foreskin

- Completely treats phimosis

- Some men can develop penile cancer associated with phimosis

- Phimosis increases penile cancer risk by five times

- Usually squamous cell carcinoma

- Usually associated with ulceration or induration

- There may also be inguinal lymphadenopathy (from concurrent infection or metastases)

- For any penile ulcer persisting more than 3 months, a biopsy should be performed to rule out squamous cell carcinoma 


\section{Figure 4. \\ Dorsal penile nerve block}

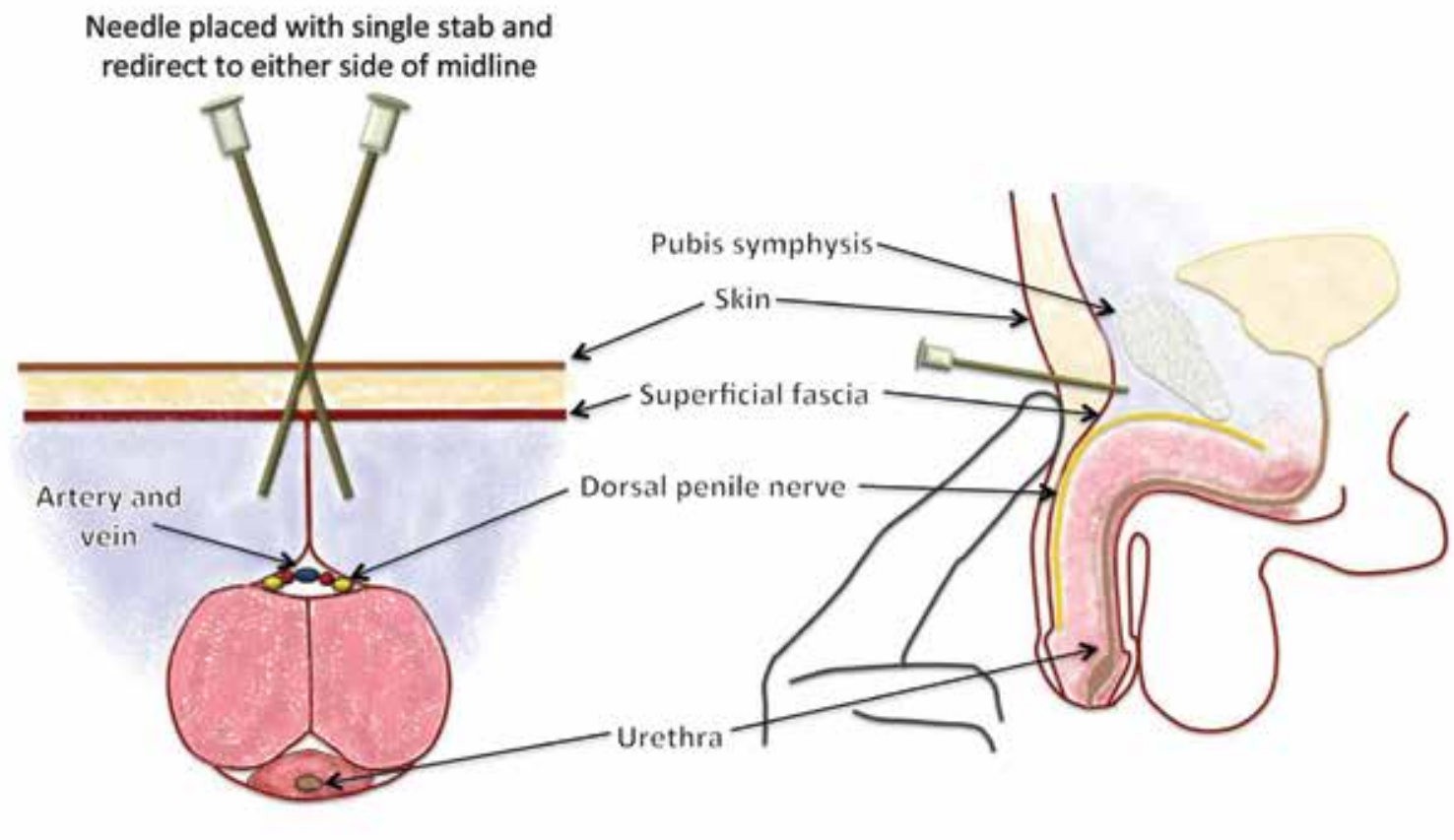

Figure 4. Dorsal penile nerve block.

Illustration by Rachel G. Khadaroo

\section{Paraphimosis}

- Occurs when the foreskin is trapped behind the glans penis and cannot be reduced (i.e., cannot be pulled back to its normal position covering the glans)

- May occur in patients with a pre-existing phimosis

- May occur iatrogenically, when a health care professional forgets to place the foreskin back after a catheter insertion or physical examination

- If it persists for hours

- It results in pain, penile edema, or even gangrene

- Therefore, it is a medical emergency

- Treatment includes compressing the glans and edema while pulling the foreskin back to its normal position; usually done with lubricant, cold compression, and local anaesthesia

- If above measures fail, an emergency dorsal slit or circumcision is required

\section{Miscellaneous Urologic Clinical Pearls}

- Not all flank pain is caused by a "kidney stone"

- Don't forget to think of ruptured abdominal aneurysm, diverticulitis, and perforated viscera
- Kidney stones cause pain by causing ureteral obstruction, so look for hydronephrosis on imaging

- Not all pyuria is a urinary tract infection (UTI)

- Urinalysis requires clinical context

- Both pyuria/bacteruria and clinical symptoms are needed for the condition to be a true UTI

- Not all scrotal swelling is a urologic problem; the scrotum also swells from generalized disease (i.e., congestive heart failure, anasarca)

- Renal ultrasonography usually overestimates the true size of a kidney stone

- Up to two-fold size miscalculations

- Also can completely miss ureteral calculi

- The ureter is not the only tubular structure in the right lower quadrant; do not forget the gonadals, appendix, etc.

- Maintain high clinical suspicion for spinal cord compression (cauda equina syndrome) in metastatic prostate cancer with new back pain, especially with lower-extremity weakness and/ or urinary retention

\section{Reference}

Wein AJ, Kavoussi LR, Novick AC et al., eds. Campbell-Walsh Urology. 9th ed. St. Louis, Mo: WB Saunders; 2007. 